\section{ON THE \\ TREATMENT OF ACUTE ORCHITIS BY PUNCTURING THE TESTICLE.}

\author{
B y HEN R Y SM T TH, EsQ., F.R.C.S., \\ ASSISTANT-SURGRON TO KING'S COLLEGE HOSPITAL.
}

Is July, 1863, a young man presented himself amongst the out-patients at King's College Hospital with gonorrbceal orchitis in a very acute form. The pain was unusually severe; and, on examining the organ, it appeared to me that suppuration had taken place, the sense of fluctuation being, as I thought, distinct. With a view of evacuating the pus, I took a bistoury, and made a free and deep incision into the supposed abscess ; but, to my astonishment and dismay, not a drop of matter escaped-only a little serum and blood. The tubes, however, of the testicle shot out, as it were, from the wound, forming a protrusion the size of a nut. Some pressure was applied by means of strips of plaster, and the patient was sent away.

Two days afterwards the man presented himself; but in a very different condition. He was quite free from pain, all the redness and most of the swelling had disappeared, and, on taking off the strapping, it was found that the protrusion of the tubes of the testis no longer existed.

This case, which was somewhat annoying to me at the time, suggested some serious reflections in reference to the speedy relief which had resulted from a practice which in reality was the effect of an error of diagnosis on my part. Was the sudden relief here a mere accident?-or, if not, to what could it be due? The quantity of serum and blood abstracted was so small that the cessation of pain and diminution of swelling could hardly be due to this cause; but it struck me forcibly that the free division of the fibrous tissue enveloping the body of the testis, and the consequent removal of tension from the organ, was the secret of the success, provided it was not a mere accident.

Influenced by this reasoning, and by the result of this case, I determined to try the effect of puncturing the testis in similar cases; and in the next case of acute orchitis which presented, I made a deep and free incision with a sharp narrow bistoury, emitting about half a teaspoonful of serum and several drachms of blood; and no other treatment beyond a little of the common aperient mixture was supplied. The result here was as successful as in the former; and as cases presented themselves, I adopted the same plan of treatment, reserving it, however, especially to those instances where the swelling and pain were very great. After the trial in a few cases, it was found that the success attending this practice was such as to lead me to adopt it as the usual treatment of acute orchitis ; and during the last twelve months I have probably treated in this way upwards of twenty cases, with results as have astonished both myself and those numerous pupils who have witnessed the practice.

In nearly erery case so treated-and I have purposely selected the most acute-the patient has experienced the most striking relief before he has left the out-patients' room; and on the next visit, forty-eight hours afterwards, the contrast presented is so remarkable that the superiority of this plan over the old fashioned modes of treatment is at once impressed forcibly upon the minds of those even who would naturally be prejudiced against so apparently heroic a treatment. The speedy subsidence of all the acute symptoms is due entirely to the puncture of the swollen and inflamed organ, for I have taken especial care not to prescribe anything else except a little of the common white mixture, or perbaps the nse of the ordinary lead lotion, and this chiefly to please the patient.

We all know what a terrible ordeal of violent remedies a patient with acute inflammation of the testicle has to undergo. In the first place, he is obliged to lie in bed for several days; a large number of leeches or the constant application of ice are necessary to relieve the pain; and at the same time the unfortunate wretch is compelled to undergo the process of severe purging and continued nausea, by repeated doses of salts and tartar emetic, before any aecided mitioation of his symptoms ensues; and two or three days mostly elapse before he recovers from the depressing influences of these several rewe.iies. Lastly, the unfortunate organ has to be submitted to the tender mercies of a dresser, who, however skilful he may be, cannot help put- ting the owner of it to severe and prolonged torture whilst he is obeying the injunctions of his superior to "strap testicle."

Now for all this I venture to subnit the plan now proposed, and one which I should call a "new" one; but it is venturing on dangerous ground to call anything new now-a-days. Moreover, my old assistant and our present house-surgeon, Mr. Richmond, informs me that when he was in Paris two years ago he saw the same method of treatment adopted there; but I never heard of it before I resorted to it, and the practice in my hands was entirely due to the accident I have related above.

Of course several of my friends and pupils have urged objections against this plan of treatment, and suggested serious results, in the form of suppuration of the organ, impairment of its function, hernia testis, and fistulons sinuses; but none of these have I witnessed. It is very natural and proper to make these objections, for we have always carefully avoided the possibility of a puncture of the testicle when using a trocar for paracentesis of the tunica vaginalis, and, indeed, I have witnessed violent suppuration of the testis speedily ensue from this accident; but it must be borne in mind that wounding of a healthy testicle with a large and blunt instrument like a trocar is a totally different thing from a careful incision made into the highly inflamed organ by a thin sharp blade.

The only inconvenient result I have witnessed from this treatment was the following :-An incision was made into the testicle of a middle-aged man, with the usual relief, but in a few days the scrotum began to swell, great pain was experienced, and the man was taken into the hospital. The objectors to the mode of treatment suggested all sorts of disasters, in the shape of suppuration of the testicle, \&c., but on careful examination it was ascertained that the swelling consisted of a large and rapid effusion of fluid into the tunica vaginalis, which was at once evacuated, with speedy relief to the patient. In another instance I made the incision much deeper than was necessary, carrying the point of the knife nearly to the back of the organ. As much as ten ounces of blood were lost, but the testis was violently inflamed and swollen, and the only effect of the accident was to make the patient somewhat faint, but at the same time to give more speedy and effectual relief than usual.

This circumstance may lead one to the belief that the relief is due solely to the escape of blood from the puncture; but this view is inconsistent with the fact that great relief is given when only a few drachms of blood, mixed with serum, are discharged. Doubtless the direct withdrawal of blood from the highly.inflamed testicle is of service, but my own view of the matter is, that the relief is in a great measure due to the withdrawal of the tension from the body of the testis by free division of the tunica albuginea.

Whatever may be the precise manner in which the good results are produced, there is no doubt of the fact, and I would earnestly suggest to surgeons, especially to my colleagues, the assistant-surgeons of the hospitals, who treat the majority of cases of orchitis, to adopt the plan proposed, rather than be submitting their patients in a routine way to all the horrors of the middle passage, from tartar emetic to strapping of the testis.

Caroline-street, Bedford-square, 1864.

P.S. - Since the above was written I have seen one of my old pupils who has been spending the last six months in the Paris hospitals, and he informs me that the ordinary practice at the Hôpital de Midi in cases of acute orchitis is to make a puncture in several places with a lancet; the instrument is not carried into the body of the testicle, but simply through the tunica albuginea. He describes the plan of treatment as most successful. - H. S.

\section{ISCHL : ITS CLITATE AND ITS BATHS.}

By DR. MI. FÜRSTENBERG,

LATE ASSISTANT-SURGEON AT THE BERTIN UXTY ERSITX YOSPITAL.

During the winter those who suffer from diseases of the chest are generally sent to a southern climate; whereas, during the warm season, many practitioners think they have sufficiently provided for these invalids if they succeed in removing them from the smoke and dust of dingy towns. It is, however, scarcely less desirable for such patients to breathe, during the summer also, a fresh, pure, moderately warm, moist air, without being exposed to the noxious influence of dust and wind. 